\title{
Nomogram Based on Systemic Immune-Inflammation Index to Predict Overall Survival in Gastric Cancer Patients
}

\author{
Hongtai Shi, ${ }^{1}$ Youqin Jiang, ${ }^{1}$ Honggang Cao, ${ }^{2}$ Haiwen $\mathrm{Zhu},{ }^{1}$ Bin Chen, ${ }^{1}$ and Weiwei Ji $\mathbb{D}^{3}$ \\ ${ }^{1}$ Department of Radiotherapy, The Third People's Hospital of Yancheng, 75 Juchang Street, Yancheng 224005, China \\ ${ }^{2}$ Department of Medical Oncology, The Third People's Hospital of Yancheng, 75 Juchang Street, Yancheng 224005, China \\ ${ }^{3}$ Department of General Surgery, The Third People's Hospital of Yancheng, 75 Juchang Street, Yancheng 224005, China
}

Correspondence should be addressed to Weiwei Ji; 450326311@qq.com

Received 14 August 2018; Accepted 1 October 2018; Published 2 December 2018

Academic Editor: Chiara Nicolazzo

Copyright (c) 2018 Hongtai Shi et al. This is an open access article distributed under the Creative Commons Attribution License, which permits unrestricted use, distribution, and reproduction in any medium, provided the original work is properly cited.

Background. The systemic immune-inflammation index (SII), based on peripheral lymphocytes, neutrophils, and platelet count, has been used as a prognostic marker for several tumors. However, use of the SII has not been reported for gastric cancer. Methods. We evaluated the prognostic value of the SII in primary and validation cohorts. We also established an effective prognostic nomogram for gastric cancer based on $\mathrm{R}$ language. The predictive accuracy and discriminative ability of the nomogram were determined using the concordance index ( $\mathrm{C}$ index) and a calibration curve and were compared with TNM classifications. Results. The Kaplan-Meier survival analysis results showed that the high SII was associated with poor prognosis of gastric cancer patients in the primary and validation cohorts. SII proved to be related to tumor location, histological grade, tumor size, TNM stage, and perineural infiltration in patients with gastric cancer and was an independent prognostic factor for patients with gastric cancer. SII has a better predictive ability than other existing prognostic indexes based on inflammation, such as NLR, PLR, and MLR. The nomogram established can accurately predict the 3 - and 5-year survival rates of patients with gastric cancer after operation, and its accuracy is significantly higher than that of the 8th edition of the AJCC staging system. Conclusion. SII can independently predict the overall survival of patients with gastric cancer after operation, which is superior to the existing systemic inflammatory indexes. The prognostic nomogram based on SII is a reliable model for predicting the postoperative survival of patients with gastric cancer.

\section{Introduction}

Gastric cancer is the fifth most common malignant tumor and the third leading cause of cancer death in the world and has become a major global health problem due to high morbidity and mortality $[1,2]$. Although the incidence and mortality of gastric cancer have declined in the last decade, it is still the third most common cause of cancer death in Chinese men and the second most common cause of cancer in Chinese women [3]. Currently, surgery is still the main treatment for gastric cancer, but the survival rate of patients with gastric cancer is lower than 30\% [4]. Recurrence or metastasis will occur in approximately $35-70 \%$ patients within 5 years, even after radical resection [5]. To reduce the risk of postoperative recurrence and metastasis, early diagnosis and developing an appropriate treatment plan based on the expected survival time of patients will help improve the cure rate of gastric cancer and the survival quality of patients. At present, clinicians mainly evaluate the prognosis of patients with gastric cancer according to the $8^{\text {th }}$ edition of the American Joint Committee on Cancer tumor node metastasis (AJCC-TNM) staging system. However, the prognosis of patients with gastric cancer within the same TNM stage is usually different after receiving similar treatment [6]. Therefore, further studies are needed to identify new tumor markers with high specificity and sensitivity in gastric cancer and to distinguish patient subgroups with a high risk of recurrence and metastasis to accurately predict the prognosis of patients with gastric cancer and determine the optimal therapeutic strategy.

Tumor-related inflammation plays an important role in the occurrence and development of tumors, and immune 
and inflammatory cells are considered important components in the tumor microenvironment [7]. Immune and inflammatory cells in peripheral blood, such as neutrophils, monocytes, platelets, and lymphocytes, are believed to lead to invasion and metastasis of tumor cells, which have close correlations with the progression of a variety of tumors [8-10]. Some indexes of the above inflammatory cells, such as the neutrophil-lymphocyte ratio (NLR), plateletlymphocyte ratio (PLR), and monocyte-lymphocyte ratio (MLR), have become prognostic factors for various cancers and are used to predict survival and recurrence of cancers, including gastric cancer [11-14]. Recently, the systemic immune-inflammation index (SII), based on peripheral lymphocytes, neutrophils, and platelet count, has been used to better reflect the balance between host inflammation and immune status; its prognostic value in hepatocellular carcinoma [15, 16], oesophageal cancer [17], colorectal cancer [18], and small cell lung cancer [19] has been confirmed, but its value in gastric cancer remains unclear. In this study, the prognostic value of SII for gastric cancer patients who underwent radical surgery was evaluated, and the prognostic nomogram of resectable gastric cancer was plotted and compared with the traditional AJCC-TNM staging system to determine whether the model can evaluate the prognosis more accurately, since the nomogram has been confirmed in other validation cohorts.

\section{Materials and Methods}

2.1. Clinical Data of Patients. A total of 688 patients with gastric cancer receiving radical resection in Fudan University Cancer Hospital from 2012 to 2014 were collected as the primary cohort. Another 174 patients with gastric cancer receiving radical resection in the Third People's Hospital of Yancheng were enrolled as the validation cohort. In this study, all patients had gastric adenocarcinoma, and others with malignant gastric tumors, such as lymphoma, gastrointestinal stromal tumors, and gastric stump carcinoma, were excluded from this study. No patients underwent neoadjuvant chemotherapy. Moreover, patients with active infection or inflammatory diseases within 1 month before blood examination were also excluded. Patients pathologically diagnosed with T3, T4, or lymph node metastasis and high-risk factors (poorly differentiated tumors, lymphatic vascular infiltration, etc.) were treated with fluorouracil-based adjuvant chemotherapy after operation. OS was determined by the period from the time of surgery to the last follow-up or date of patient death. In the primary cohort, the median follow-up time was 36 months (1-75 months), and the number of events for OS was 383 at the last follow-up. In the validation cohort, the median follow-up time was 32 months (4-69 months), and the number of events for OS was 86 at the last follow-up. All patients signed the informed consent, all studies were performed according to the Helsinki Declaration, and this retrospective experiment was approved by the Ethics Committee of the Third People's Hospital of Yancheng.

2.2. Systemic Inflammatory Indexes. Peripheral blood was collected within 1 week before the operation to detect
TABLE 1: Clinicopathological characteristics of patients with gastric cancer in the primary cohort and the validation cohort.

\begin{tabular}{|c|c|c|c|c|}
\hline \multirow[t]{2}{*}{ Characteristic } & \multicolumn{2}{|c|}{$\begin{array}{c}\text { Primary } \\
\text { cohort } \\
(n=688)\end{array}$} & \multicolumn{2}{|c|}{$\begin{array}{c}\text { Validation } \\
\text { cohort } \\
(n=174)\end{array}$} \\
\hline & $\begin{array}{c}\text { No. of } \\
\text { patients }\end{array}$ & $\%$ & $\begin{array}{c}\text { No. of } \\
\text { patients }\end{array}$ & $\%$ \\
\hline \multicolumn{5}{|l|}{ Sex } \\
\hline Male & 471 & 68.5 & 131 & 75.3 \\
\hline Female & 217 & 31.5 & 43 & 24.7 \\
\hline \multicolumn{5}{|l|}{ Age } \\
\hline$\leq 60$ & 463 & 67.3 & 103 & 59.2 \\
\hline$>60$ & 225 & 32.7 & 71 & 40.8 \\
\hline \multicolumn{5}{|l|}{ Tumor location } \\
\hline Upper & 106 & 15.4 & 28 & 16.1 \\
\hline Middle & 267 & 38.8 & 53 & 30.5 \\
\hline Lower & 315 & 45.8 & 93 & 53.4 \\
\hline \multicolumn{5}{|l|}{ Histological grade } \\
\hline Well or moderately differentiated & 367 & 53.3 & 100 & 57.5 \\
\hline Poorly or not differentiated & 321 & 46.7 & 74 & 42.5 \\
\hline \multicolumn{5}{|l|}{ Lauren type } \\
\hline Diffuse & 115 & 16.7 & 34 & 19.5 \\
\hline Intestinal & 328 & 47.7 & 75 & 43.1 \\
\hline Mixed & 245 & 35.6 & 65 & 37.4 \\
\hline \multicolumn{5}{|l|}{ Tumor size } \\
\hline$\leq 5$ & 316 & 45.9 & 72 & 41.4 \\
\hline$>5$ & 372 & 54.1 & 102 & 58.6 \\
\hline \multicolumn{5}{|l|}{ Lymphovascular invasion } \\
\hline No & 483 & 70.2 & 111 & 63.8 \\
\hline Yes & 205 & 29.8 & 63 & 36.2 \\
\hline \multicolumn{5}{|l|}{ Perineural invasion } \\
\hline No & 392 & 57.0 & 122 & 29.9 \\
\hline Yes & 296 & 43.0 & 52 & 70.1 \\
\hline \multicolumn{5}{|l|}{ TNM stage (AJCC, 8th) } \\
\hline I & 183 & 26.6 & 43 & 24.7 \\
\hline II & 215 & 31.3 & 88 & 50.6 \\
\hline III & 290 & 42.2 & 43 & 24.7 \\
\hline \multicolumn{5}{|l|}{ Adjuvant chemotherapy } \\
\hline No & 267 & 38.8 & 62 & 35.6 \\
\hline Yes & 421 & 61.2 & 112 & 64.4 \\
\hline
\end{tabular}

neutrophils, lymphocytes, and platelet count. Blood cell counts were detected by Sysmex XT-1800i Automated Hematology System (Shanghai, China): NLR=neutrophil count/lymphocyte count; PLR = platelet count/lymphocyte count; MLR = monocyte count/lymphocyte count; SII = platelet count $\times$ neutrophil count/lymphocyte count. The optimal cut-off values for the above indexes were obtained using X-tile software (https://x-tile.software.informer.com/) [20]: SII (SII $\leq 320$, SII $>320)$, NLR $(\mathrm{NLR} \leq 1.3$, NLR $>1.3)$, $\operatorname{PLR}(\mathrm{PLR} \leq 135, \operatorname{PLR}>135)$, and $\operatorname{MLR} \quad(\mathrm{MLR} \leq 0.23$, NLR > 0.23). 
TABLE 2: Baseline characteristics for patients with SII $\leq 320$ versus SII $>320$ in primary and validation cohorts.

\begin{tabular}{|c|c|c|c|c|c|c|c|c|}
\hline \multirow{2}{*}{ Clinical parameter } & \multicolumn{4}{|c|}{ Primary cohort } & \multicolumn{4}{|c|}{ Validation cohort } \\
\hline & $\mathrm{SII} \leq 320(356)$ & SII > $320(332)$ & $\chi^{2}$ & $P$ & $\mathrm{SII} \leq 320(90)$ & SII $>320(84)$ & $\chi^{2}$ & $P$ \\
\hline Sex & & & 0.75 & 0.386 & & & 0.07 & 0.79 \\
\hline Male & 249 & 222 & & & 67 & 64 & & \\
\hline Female & 107 & 110 & & & 23 & 20 & & \\
\hline Age & & & 0.55 & 0.457 & & & 0.01 & 0.932 \\
\hline$\leq 60$ & 235 & 228 & & & 53 & 50 & & \\
\hline$>60$ & 121 & 104 & & & 37 & 34 & & \\
\hline Tumor location & & & 16.51 & $<0.001^{*}$ & & & 1.35 & 0.51 \\
\hline Upper & 36 & 70 & & & 17 & 11 & & \\
\hline Middle & 142 & 125 & & & 25 & 28 & & \\
\hline Lower & 178 & 137 & & & 48 & 45 & & \\
\hline Histological grade & & & 31.85 & $<0.001^{*}$ & & & 8.10 & $0.004^{*}$ \\
\hline Well or moderately differentiated & 153 & 214 & & & 61 & 39 & & \\
\hline Poorly or not differentiated & 203 & 118 & & & 29 & 45 & & \\
\hline Lauren type & & & 2.15 & 0.341 & & & 3.05 & 0.218 \\
\hline Diffuse & 65 & 50 & & & 17 & 17 & & \\
\hline Intestinal & 161 & 167 & & & 34 & 41 & & \\
\hline Mixed & 130 & 115 & & & 39 & 26 & & \\
\hline Tumor size & & & 55.11 & $<0.001^{*}$ & & & 13.50 & $<0.001^{*}$ \\
\hline$\leq 5$ & 212 & 104 & & & 45 & 27 & & \\
\hline$>5$ & 144 & 228 & & & 35 & 67 & & \\
\hline Lymphovascular invasion & & & 1.39 & 0.238 & & & 0.02 & 0.896 \\
\hline No & 257 & 226 & & & 57 & 54 & & \\
\hline Yes & 99 & 106 & & & 33 & 30 & & \\
\hline Perineural invasion & & & 6.20 & $0.013^{*}$ & & & 0.09 & 0.766 \\
\hline No & 219 & 173 & & & 64 & 58 & & \\
\hline Yes & 137 & 159 & & & 26 & 26 & & \\
\hline TNM stage (AJCC, 8th) & & & 83.59 & $<0.001^{*}$ & & & 13.99 & $0.001^{*}$ \\
\hline I & 119 & 64 & & & 26 & 17 & & \\
\hline II & 146 & 69 & & & 35 & 53 & & \\
\hline III & 91 & 199 & & & 9 & 34 & & \\
\hline Adjuvant chemotherapy & & & 0.84 & 0.36 & & & 1.55 & 0.213 \\
\hline No & 144 & 123 & & & 36 & 26 & & \\
\hline Yes & 212 & 209 & & & 54 & 58 & & \\
\hline NLR & & & 140.83 & $<0.001^{*}$ & & & 48.38 & $<0.001^{*}$ \\
\hline $\mathrm{NLR} \leq 1.3$ & 268 & 100 & & & 51 & 6 & & \\
\hline $\mathrm{NLR}>1.3$ & 88 & 232 & & & 39 & 78 & & \\
\hline PLR & & & 123.64 & $<0.001^{*}$ & & & 103.24 & $<0.001^{*}$ \\
\hline $\mathrm{PLR} \leq 135$ & 212 & 60 & & & 82 & 12 & & \\
\hline PLR $>135$ & 144 & 272 & & & 8 & 72 & & \\
\hline MLR & & & 38.74 & $<0.001^{*}$ & & & 21.60 & $<0.001^{*}$ \\
\hline MLR $\leq 0.23$ & 226 & 132 & & & 78 & 46 & & \\
\hline MLR $>0.23$ & 130 & 200 & & & 12 & 38 & & \\
\hline
\end{tabular}

2.3. Statistical Analysis. SPSS 22.0 software, GraphPad Prism 5.0 , and $\mathrm{R}$ language were used for statistical analysis. A time-dependent ROC curve was carried out using $\mathrm{R}$ software version 3.2.0 (http://www.r-project.org/) with rms and survival ROC packages. Analysis of variance and the Pearson chi-square test were used to evaluate the correlations among variables. The survival curve was plotted using the Kaplan-Meier method, and differences between groups 

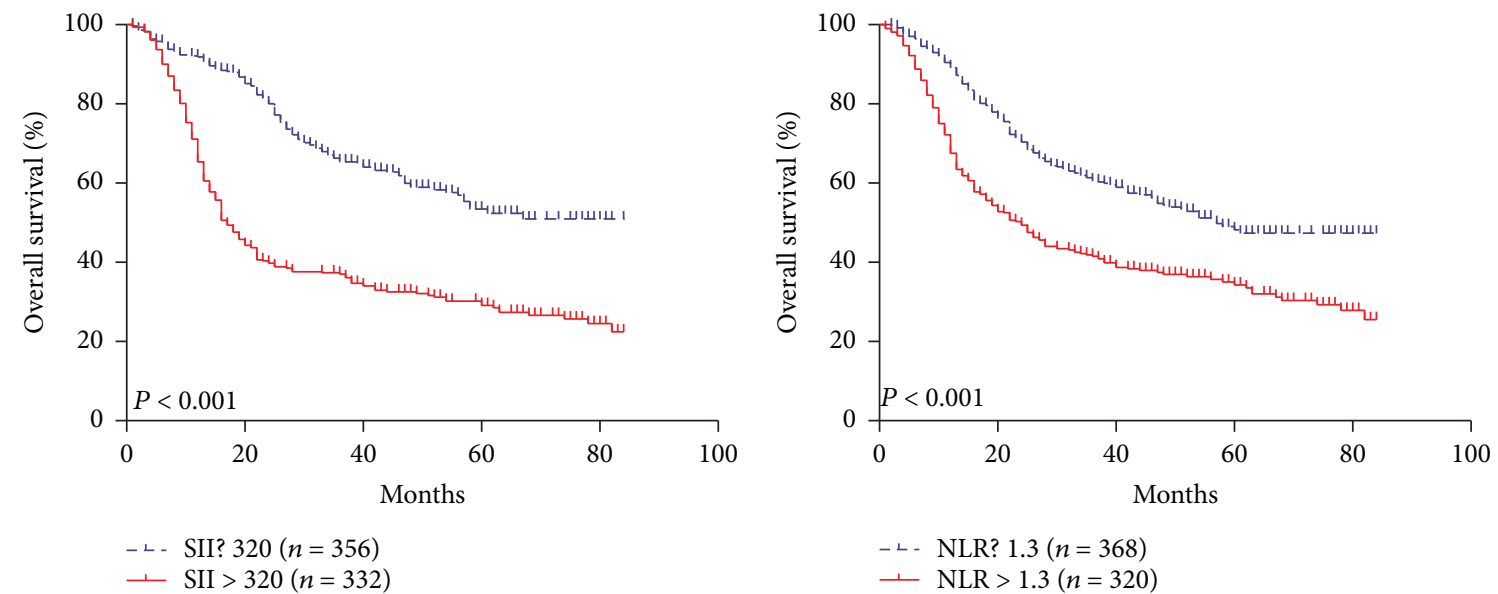

(a)
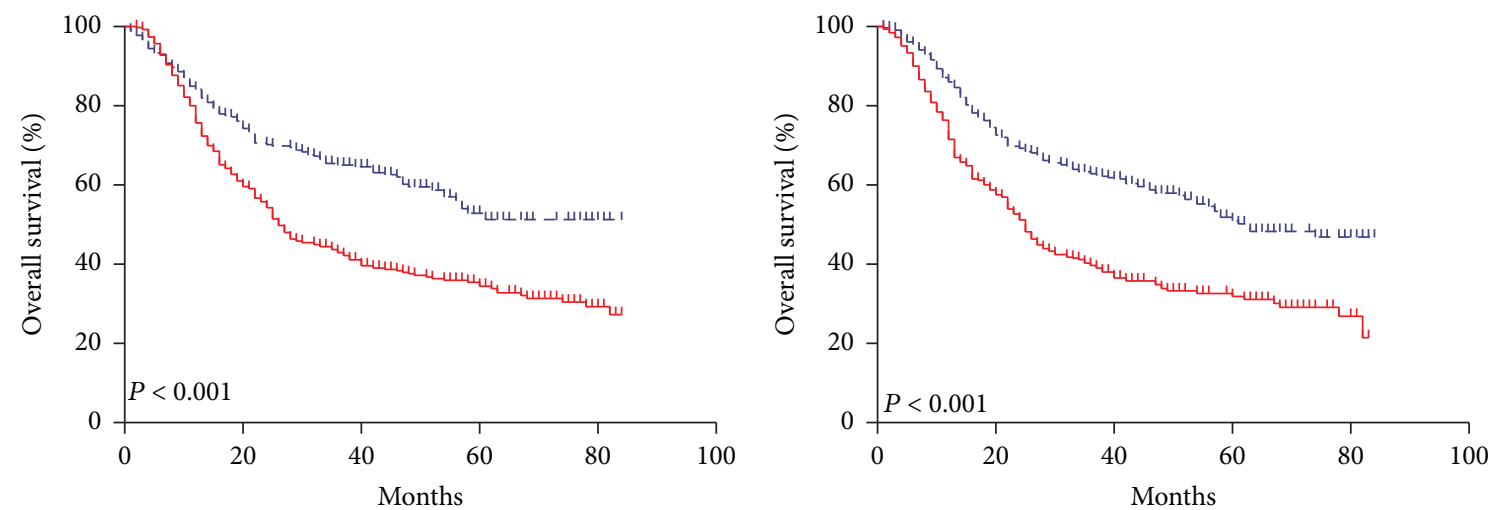

- L. PLR? $135(n=272)$

$\perp$ PLR $>135(n=416)$

- ᄂ - MLR? $0.23(n=358)$

$+\operatorname{MLR}>0.23(n=330)$

(c)
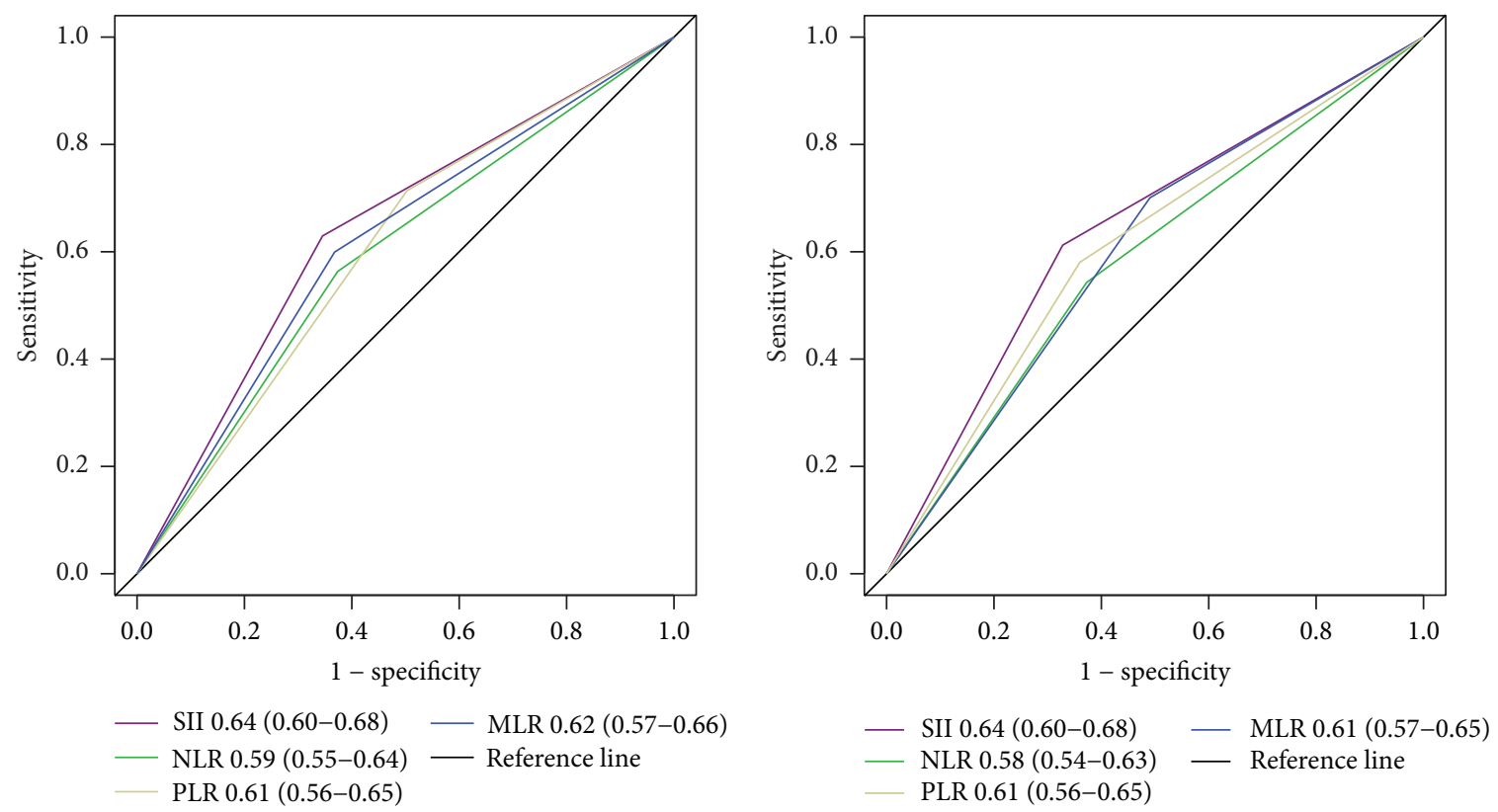

— SII $0.64(0.60-0.68) \quad$ - MLR $0.62(0.57-0.66)$

— NLR $0.59(0.55-0.64)$ — Reference line

PLR $0.61(0.56-0.65)$

(f)

FIGURE 1: The prognostic significance of the SII (a), NLR (b), PLR (c), and MLR (d) in gastric cancer in the primary cohort. Predictive ability of the SII in gastric cancer was compared with PLR, NLR, and MLR by ROC curves in 3 years (e) and 5 years (f) in the primary cohort. 
TABLE 3: Univariate and multivariate Cox regression analyses for overall survival in patients with gastric cancer.

\begin{tabular}{|c|c|c|c|c|}
\hline \multirow{2}{*}{ Variables } & \multicolumn{2}{|c|}{ Univariate analysis } & \multicolumn{2}{|c|}{ Multivariate analysis } \\
\hline & HR (95\% CI) & $P$ value & HR (95\% CI) & $P$ value \\
\hline \multicolumn{5}{|l|}{ Primary cohort } \\
\hline Sex: male vs. female & $1.14(0.92-1.41)$ & 0.223 & & \\
\hline Age: $>60$ vs. $\leq 60$ & $1.24(0.99-1.54)$ & 0.053 & & \\
\hline Tumor location & & $0.001^{*}$ & & 0.057 \\
\hline Middle vs. upper & $0.71(0.54-0.93)$ & $0.018^{*}$ & $0.84(0.63-1.12)$ & 0.227 \\
\hline Lower vs. upper & $0.58(0.44-0.76)$ & $<0.001^{*}$ & $0.71(0.53-0.95)$ & $0.020^{*}$ \\
\hline Grade: poorly vs. well & $0.56(0.40-0.72)$ & $<0.001^{*}$ & $1.12(0.89-1.41)$ & 0.350 \\
\hline Lauren type & & 0.137 & & \\
\hline Intestinal vs. diffuse & $1.21(0.91-1.62)$ & 0.195 & & \\
\hline Mixed vs. diffuse & $0.98(0.72-1.34)$ & 0.907 & & \\
\hline Tumor size: $>5$ vs. $\leq 5$ & $2.38(1.92-2.94)$ & $<0.001^{*}$ & $1.19(0.94-1.51)$ & 0.142 \\
\hline Lymphovascular: yes vs. no & $1.21(0.97-1.50)$ & 0.086 & & \\
\hline Perineural: yes vs. no & $1.58(1.29-1.93)$ & $<0.001^{*}$ & $1.21(0.96-1.53)$ & 0.101 \\
\hline TNM stage & & $<0.001^{*}$ & & $<0.001^{*}$ \\
\hline II vs. I & $2.03(1.46-2.83)$ & $<0.001^{*}$ & $1.77(1.26-2.47)$ & $0.001^{*}$ \\
\hline III vs. I & $4.68(3.46-6.34)$ & $<0.001^{*}$ & $3.10(2.27-4.24)$ & $<0.001^{*}$ \\
\hline Chemotherapy: Yes vs. No & $1.12(0.91-1.37)$ & 0.282 & & \\
\hline SII: $>320$ vs. $\leq 320$ & $2.47(2.01-3.04)$ & $<0.001^{*}$ & $1.61(1.27-2.05)$ & $0.041^{*}$ \\
\hline NLR: $>1.3$ vs. $\leq 1.3$ & $1.79(1.47-2.20)$ & $<0.001^{*}$ & $1.25(0.99-1.58)$ & 0.054 \\
\hline PLR: >135 vs. $\leq 135$ & $1.82(1.46-2.27)$ & $<0.001^{*}$ & $1.04(0.81-1.33)$ & 0.787 \\
\hline MLR: $>0.23$ vs. $\leq 0.23$ & $1.88(1.54-2.31)$ & $<0.001^{*}$ & $1.17(0.93-1.47)$ & 0.183 \\
\hline \multicolumn{5}{|l|}{ Validation cohort } \\
\hline Sex: male vs. female & $1.01(0.62-1.63)$ & 0.987 & & \\
\hline Age: $>60$ vs. $\leq 60$ & $1.09(0.88-1.36)$ & 0.442 & & \\
\hline Tumor location & & $0.046^{*}$ & & 0.098 \\
\hline Middle vs. upper & $0.51(0.29-0.89)$ & $0.018^{*}$ & $0.61(0.30-1.41)$ & 0.146 \\
\hline Lower vs. upper & $0.51(0.27-0.94)$ & $0.032^{*}$ & $0.65(0.38-1.10)$ & 0.087 \\
\hline Grade: poorly vs. well & $1.25(1.01-1.54)$ & $0.041^{*}$ & $1.48(0.95-2.29)$ & 0.080 \\
\hline Lauren type & & 0.386 & & \\
\hline Intestinal vs. diffuse & $0.65(0.34-1.25)$ & 0.196 & & \\
\hline Mixed vs. diffuse & $0.99(0.63-1.57)$ & 0.979 & & \\
\hline Tumor size: $>5$ vs. $\leq 5$ & $1.72(1.09-2.70)$ & $0.019^{*}$ & $1.81(0.81-4.04)$ & 0.151 \\
\hline Lymphovascular: yes vs. no & $1.48(0.90-2.43)$ & 0.118 & & \\
\hline Perineural: yes vs. no & $1.70(1.06-2.72)$ & $0.028^{*}$ & $1.43(0.61-3.39)$ & 0.414 \\
\hline TNM stage & & $<0.001^{*}$ & & $<0.001^{*}$ \\
\hline II vs. I & $2.91(1.45-5.83)$ & $0.003^{*}$ & $3.19(1.43-3.63)$ & $0.002^{*}$ \\
\hline III vs. I & $7.12(3.50-14.50)$ & $<0.001^{*}$ & $7.05(3.15-15.81)$ & $<0.001^{*}$ \\
\hline Chemotherapy: yes vs. no & $1.26(0.79-2.01)$ & $0.001^{*}$ & & \\
\hline SII: $>320$ vs. $\leq 320$ & $2.75(1.75-4.32)$ & $<0.001^{*}$ & $2.94(1.83-4.73)$ & $<0.001^{*}$ \\
\hline NLR: $>1.3$ vs. $\leq 1.3$ & $2.03(1.23-3.35)$ & $0.006^{*}$ & $1.71(0.96-3.39)$ & 0.067 \\
\hline PLR: $>135$ vs. $\leq 135$ & $1.90(1.24-2.94)$ & $0.004^{*}$ & $1.46(0.93-2.30)$ & 0.102 \\
\hline MLR: $>0.23$ vs. $\leq 0.23$ & $1.56(1.02-2.40)$ & $0.046^{*}$ & $1.22(0.76-1.95)$ & 0.420 \\
\hline
\end{tabular}



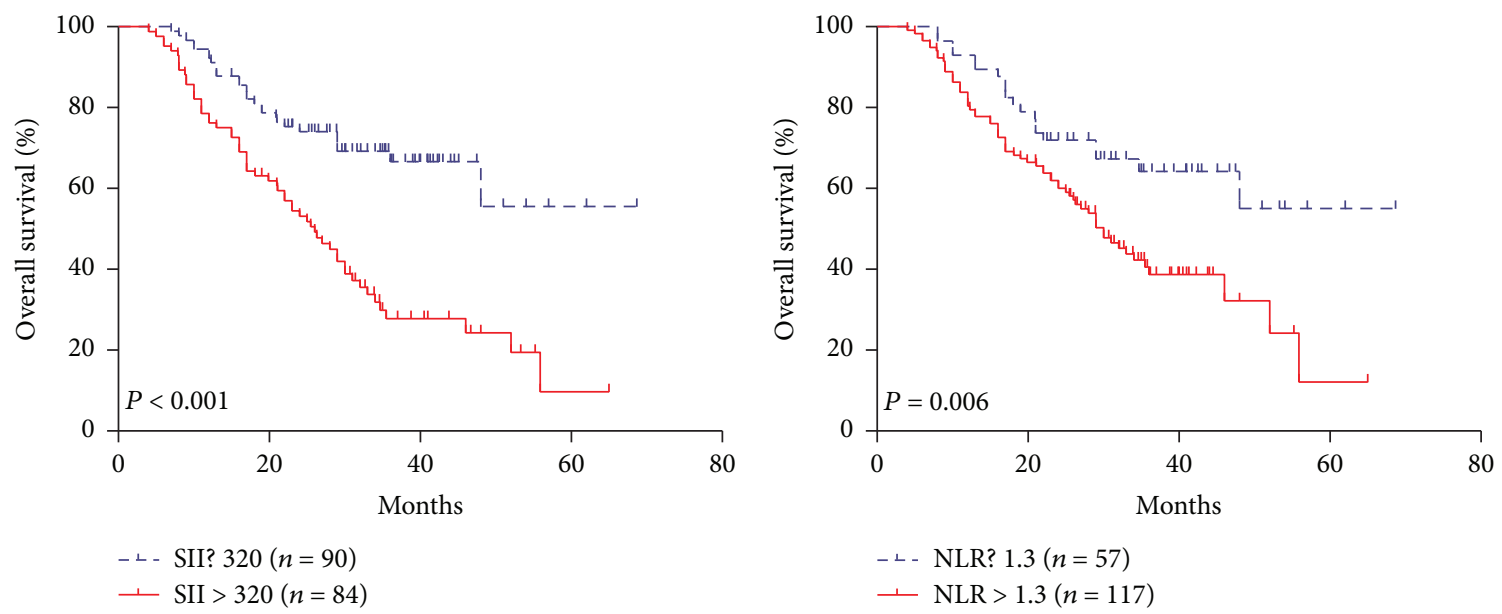

(a)
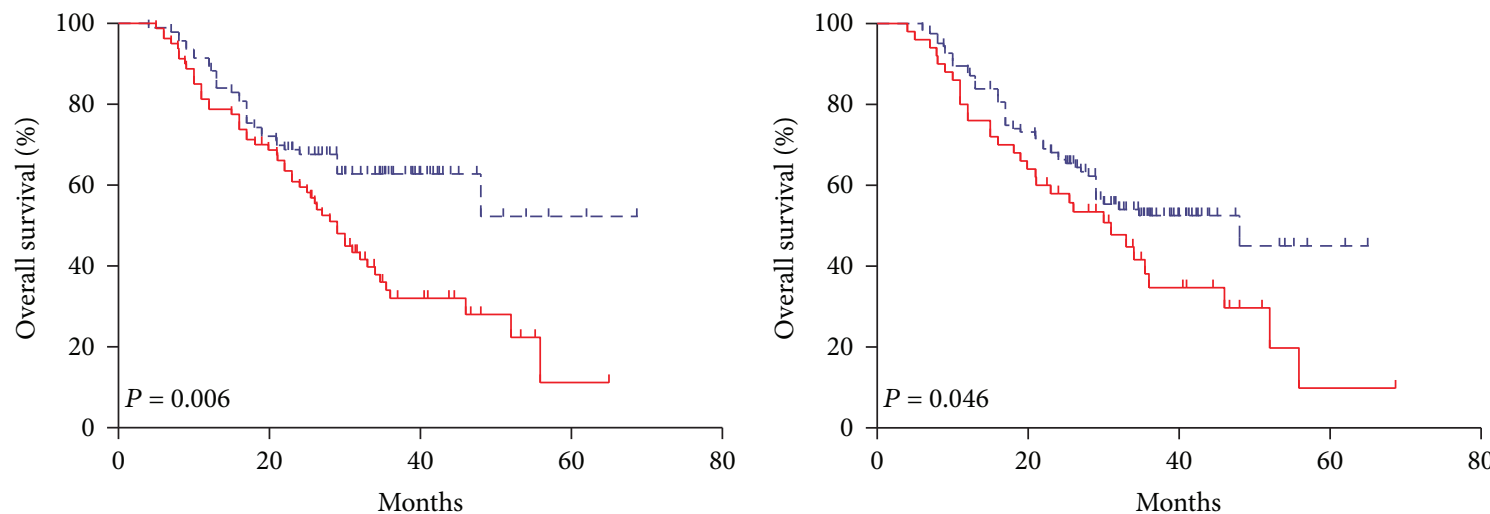

$$
\begin{aligned}
& \text { - L } \text { PLR? } 135(n=94) \\
& \text { - } \operatorname{PLR}>135(n=80)
\end{aligned}
$$$$
\text { - - - MLR? } 0.23(n=124)
$$$$
-\operatorname{MLR}>0.23(n=50)
$$

(c)

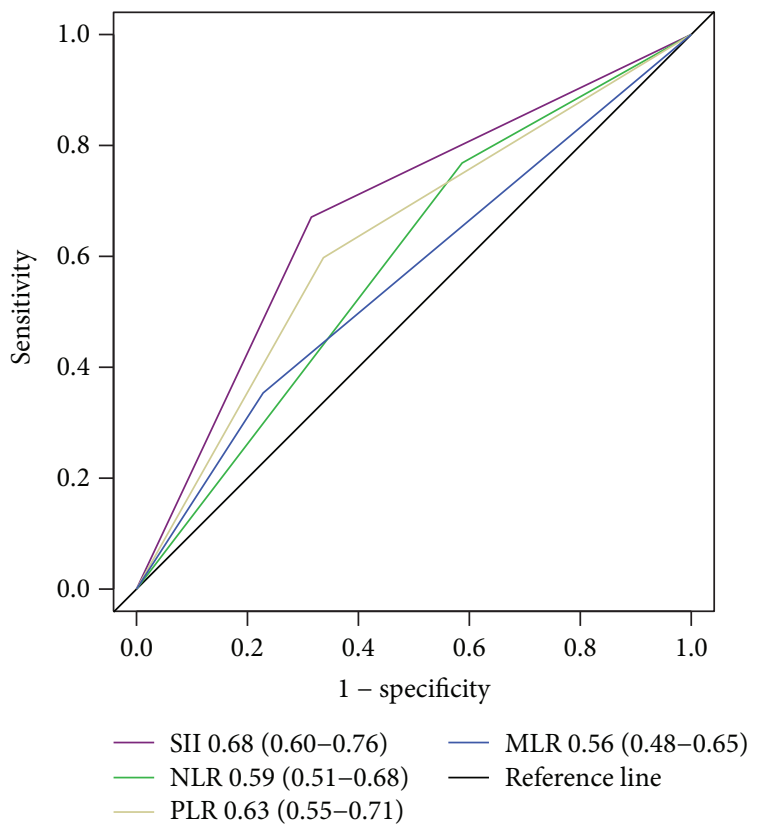

(e)

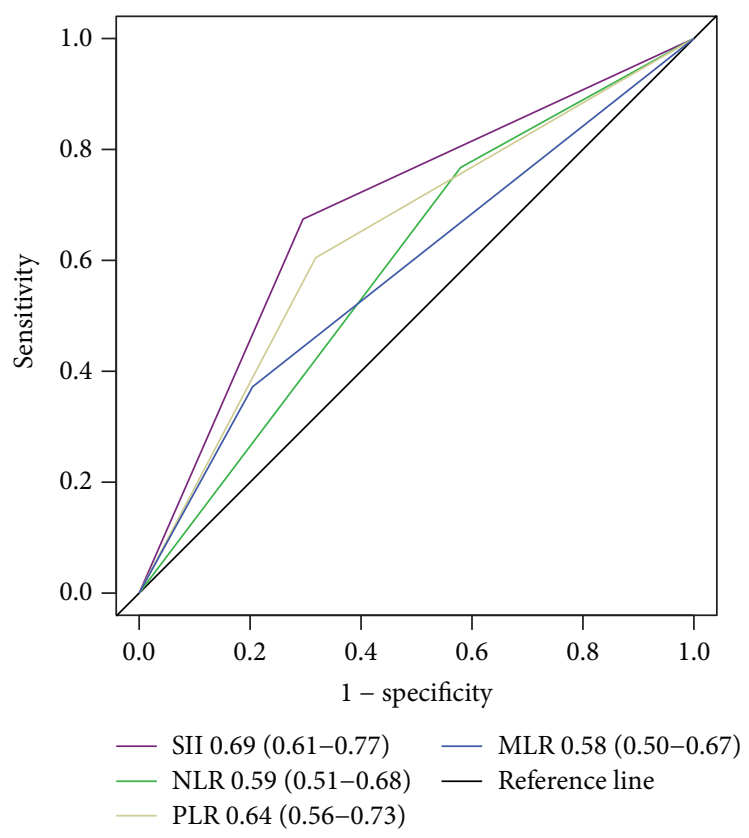

(f)

FIGURE 2: The prognostic significance of the SII (a), NLR (b), PLR (c), and MLR (d) in gastric cancer in the validation cohort. Predictive ability of the SII in gastric cancer was compared with PLR, NLR, and MLR by ROC curves in 3 years (e) and 5 years (f) in the validation cohort. 


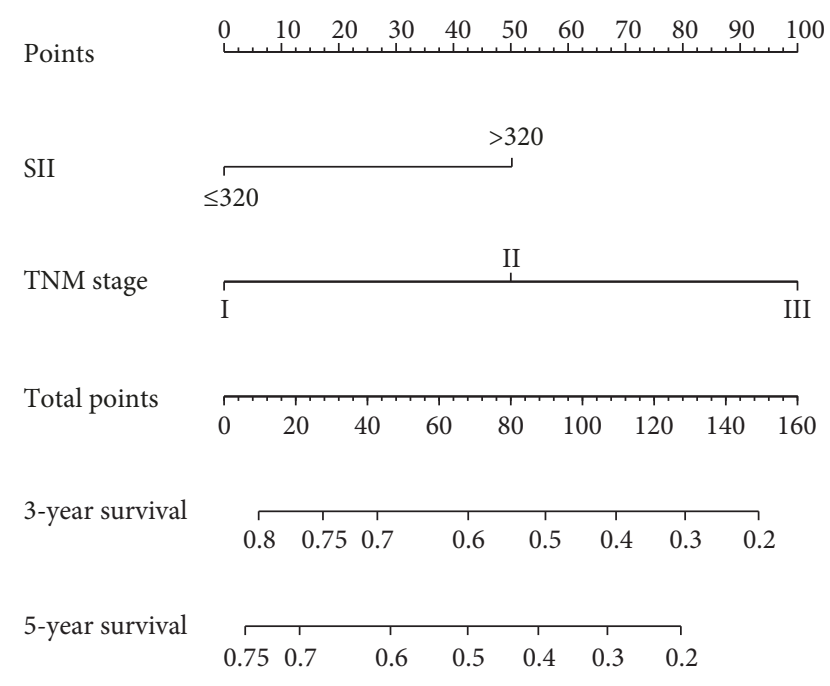

FIGURE 3: Evaluation of nomogram-integrated SII and TNM stage in patients with gastric cancer.

were compared via the log-rank test. Univariate and multivariate Cox proportional hazard models were adopted to determine prognostic indexes, and the nomogram was plotted based on multivariate analysis results. Moreover, the efficiency of the nomogram was evaluated, and the timedependent receiver operating characteristics (ROC) curve and $\mathrm{C}$ index were analysed to compare the discriminatory ability of different models in total survival. Unless otherwise specified, $P<0.05$ suggested that the difference was statistically significant.

\section{Results}

3.1. Clinicopathological Features of the Patient. In the primary cohort, there were 471 males and 217 females, aged 56 years on average, including 316 cases of tumor diameter $\leq 5 \mathrm{~cm}$ and 372 cases of tumor diameter $>5 \mathrm{~cm}$. There were 367 cases of high-moderate differentiation, and 321 cases of poor differentiation or no differentiation. In terms of the Lauren type, there were 328 cases of intestinal type, 115 cases of diffuse type, and 245 cases of mixed type. According to the 8th edition of the AJCC-TNM staging system, there were 183 cases in stage I, 215 cases in stage II, and 290 cases in stage III. Four hundred twenty-one patients underwent postoperative adjuvant chemotherapy, while 267 patients underwent no postoperative adjuvant chemotherapy. Other clinicopathological parameters and the validation cohort of patients are shown in Table 1.

The correlation between various systemic inflammatory indexes and clinicopathological features is shown in Table 2 . In the primary cohort, the preoperative SII $>320$ was related to poor differentiation, upper tumor site, larger tumor, and later TNM stage. In the validation cohort, SII was also related to poor differentiation, larger tumor, and later TNM stage. Moreover, SII had strong correlations with other systemic inflammatory indexes (NLR, PLR, and MLR) in both the primary cohort and the validation cohort (Table 2).
3.2. Survival Analysis. In the primary cohort, the KaplanMeier survival analysis revealed that gastric cancer patients with high SII, PLR, NLR, and MLR scores had poor prognosis (Figures 1(a)-1(d)), but the counts for neutrophils, lymphocytes, platelets, and monocyte alone showed no significant influences on prognosis. The relationships between survival and tumor site, histological grade, tumor size, nerve infiltration, and TNM staging were determined according to the Cox univariate analyses. SII, PLR, NLR, and MLR were factors affecting prognosis, while gender, age, Lauren type, vascular infiltration, and postoperative adjuvant chemotherapy had no significant influences on prognosis (Table 3 ). Multivariate analysis further revealed that TNM stage and SII were independent risk factors for gastric cancer (Table 3). Among SII, NLR, PLR, and MLR, only SII was an independent risk factor for OS (HR $=1.61,95 \%$ CI: $1.27-$ $2.05, P=0.041)$. In addition, the ROC curve showed that the area under the curve of SII was larger than that of NLR, PLR, and MLR, indicating that SII is superior to other inflammatory indexes in predicting the 3- and 5-year survival rates of patients with gastric cancer (Figures $1(\mathrm{e})-1(\mathrm{f})$ ). Survival analysis of the validation cohort showed that patients with SII $\leq 320$ had longer OS, and similar results were obtained in NLR, PLR, and MLR (Figures 2(a)-2(d)). Among SII, NLR, PLR, and MLR, however, only SII was an independent prognostic index for patients with gastric cancer in the multivariate analysis $(\mathrm{HR}=2.94,95 \% \mathrm{CI}$ : $1.83-4.17$, $P<0.001$, Table 3). The ROC curve showed that the area under the curve of SII was larger than that of NLR, PLR, and MLR (Figures 2(e)-2(f)). To sum up, it is believed that SII is superior to other inflammatory indexes in predicting the 3-and 5-year survival rates of patients with gastric cancer.

3.3. Establishment and Verification of the Nomogram. Based on the multivariate analysis results, the following variables were eventually integrated in the nomogram to predict the 3- and 5-year survival of the primary cohort: TNM stage and SII (Figure 3). The C index of nomogram was 0.74 , which was significantly higher than that of TNM staging $0.70(P<0.001)$. The 3 - and 5 -year survival probability calibration charts showed that the predictive height of the nomogram was consistent with the actual observations (Figures 4(a) and 4(b)). Moreover, the nomogram predicted the survival of the primary cohort more accurately. The area under the ROC curve of the nomogram was significantly larger than that of TNM stage (Figures 4(c) and 4(d)) $(P<0.001)$.

Finally, the established nomogram was used to verify survival in the validation cohort. The calibration curve showed that the 3- and 5-year survival rates predicted by the nomogram were consistent with the actual observation (Figures 4(e) and 4(f)). The $\mathrm{C}$ index of the nomogram was 0.72 , which was significantly higher than that of TNM staging $0.69(P<0.001)$. The area under the ROC curve of the nomogram in the predictive validation cohort was also significantly larger than that of the TNM stage (Figures 4(g) and $4(\mathrm{~h}))(P<0.001)$. The above results indicate that the established nomogram, based on SII, is superior to the 


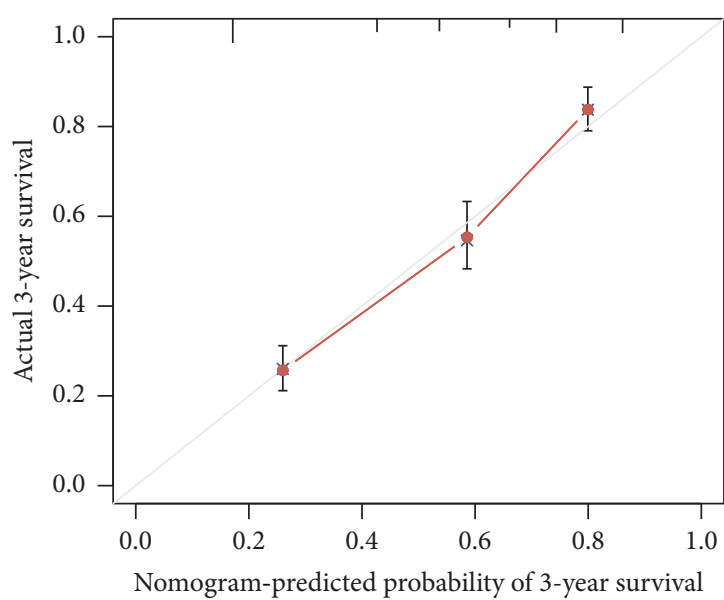

(a)

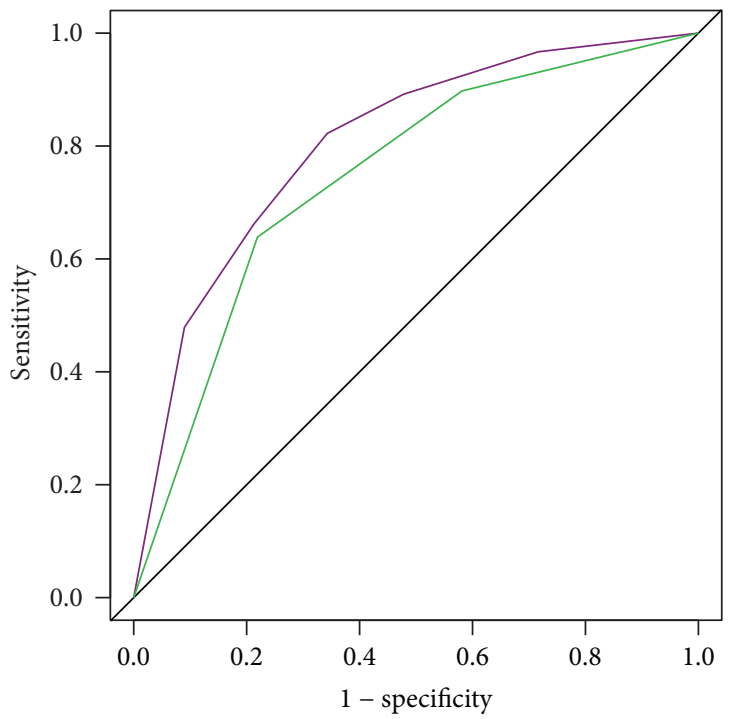

— Nomogram 0.80

— TNM stage 0.75

- Reference line

(c)

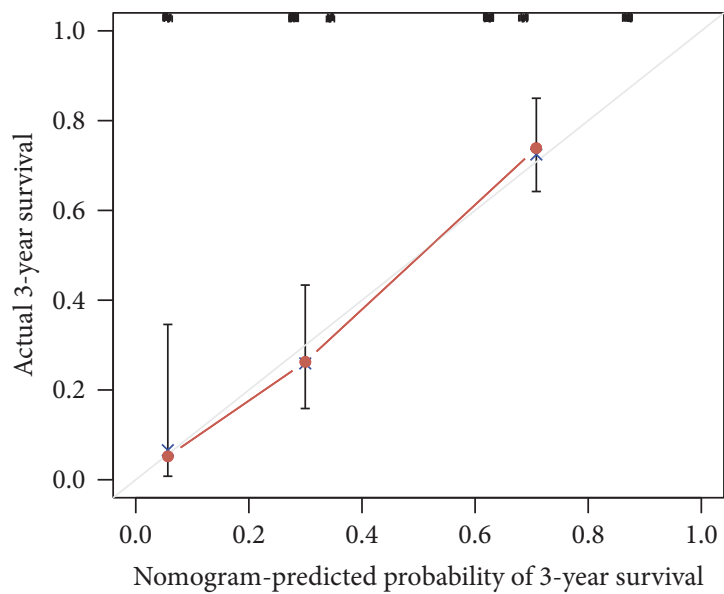

(e)

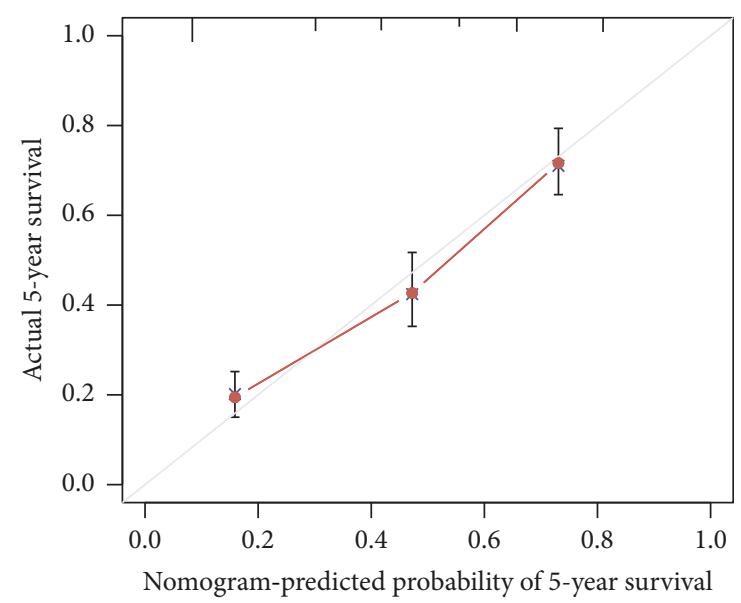

(b)

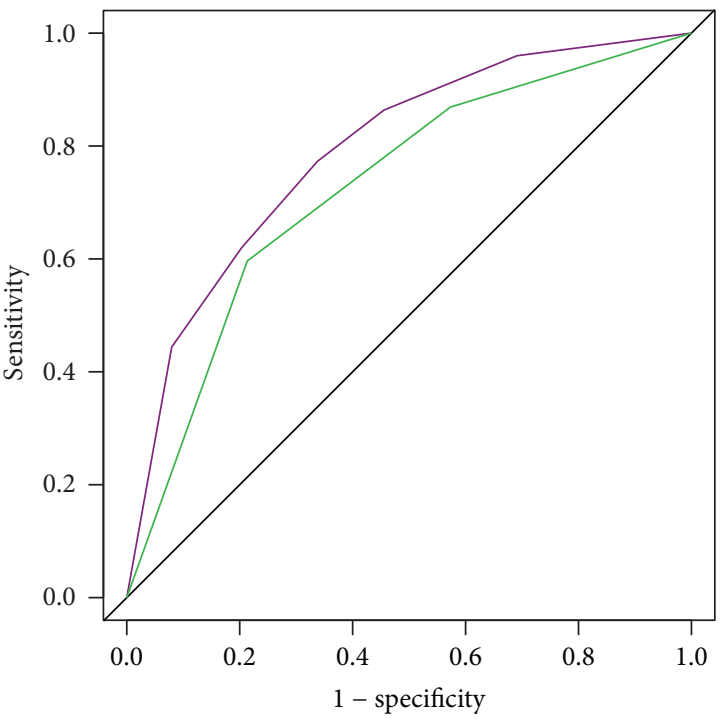

— Nomogram 0.79

— TNM stage 0.73

- Reference line

(d)

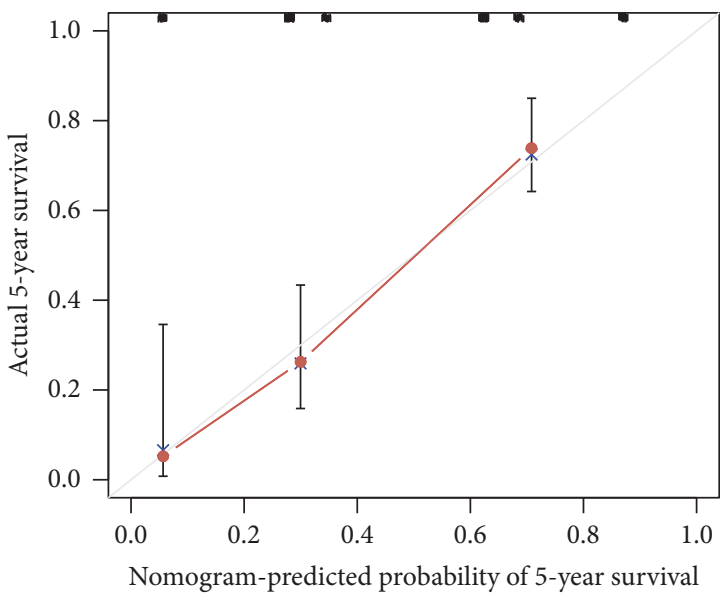

(f)

FIgUre 4: Continued. 


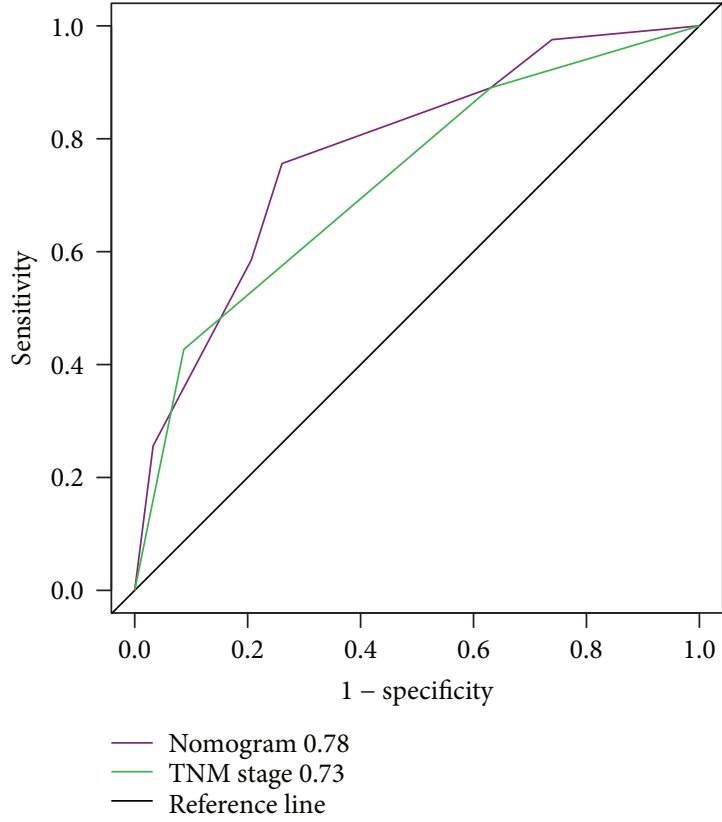

(g)

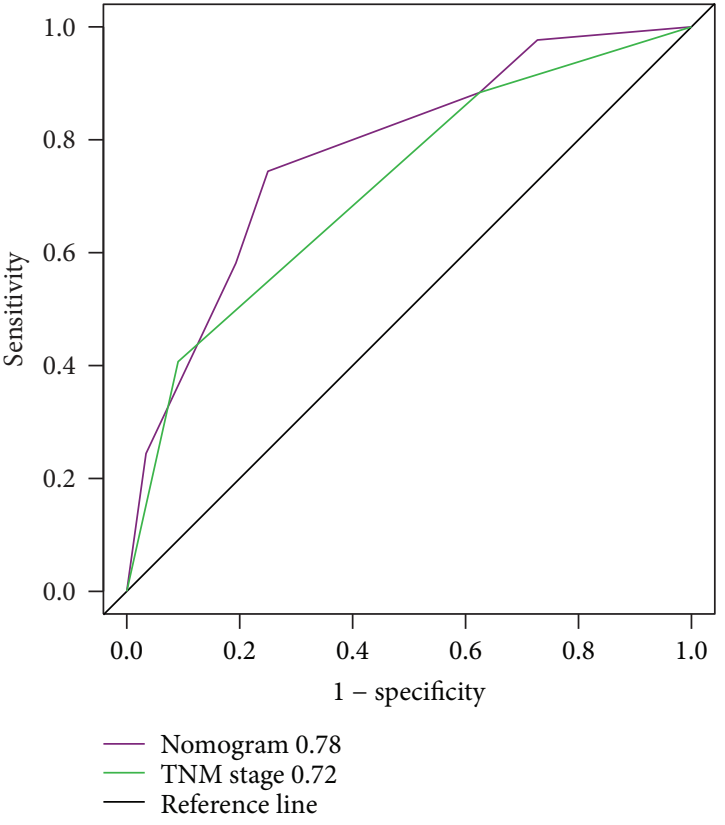

(h)

FIgURE 4: The calibration curve for predicting patient survival at 3 years (a) and 5 years (b) in the primary cohort. Time-dependent ROC curves by nomogram and TNM staging for 3-year (c) and 5-year (d) OS in the validation cohort. The calibration curve for predicting patient survival at 3 years (e) and 5 years (f) in the primary cohort. Time-dependent ROC curves by nomogram and TNM staging for 3 -year (g) and 5-year (h) OS in the validation cohort.

TNM staging system in predicting the survival of patients with gastric cancer.

\section{Discussion}

In the $19^{\text {th }}$ century, Rudolf Vichow et al., German pathologists, found leukocytes in tumor tissues and proposed that there was a close correlation between inflammation and tumors [21]. With continuing research, the important correlation between inflammation and tumors has been gradually confirmed by related epidemiological and molecular biology research. According to epidemiological surveys, major clinical evidence for the connection between chronic inflammation and tumors exists. Molecular biological studies distinguish the interaction between inflammation and tumors from phenomenon and mechanisms. Tumorrelated inflammatory factors include not only inflammatory factors produced by tumor cells but also tumor-related inflammatory cells and inflammatory factors released during tissue engineering, repair, and angiogenesis [10]. Under the status of injury or infection, the local immune system activates a large number of inflammatory cells, such as macrophages, neutrophils, mast cells, and lymphocytes. These inflammatory cells secrete a variety of cytokines, such as tumor necrosis factor (TNF), IL-6, vascular endothelial growth factor, fibroblast growth factor, platelet-derived growth factor, and extracellular matrix proteins, such as matrix metalloproteinase (MMP), elastase, neutral protease, and collagenase, forming the inflammatory microenvironment and repairing damaged tissues. However, when there is such an inflammatory microenvironment in tumor patients, a large number of inflammatory mediators that can change the stable internal environment will be released, leading to an inflammation-related cascade, tissue atrophy and destruction, and promoting malignant progression of tumors $[22,23]$. The persistent inflammatory microenvironment induces tumorigenesis, and the formation and development of tumors further aggravate the inflammatory response. Therefore, systemic inflammatory response has a clear correlation with tumor prognosis, and the prognostic scoring system based on systemic inflammatory response can effectively evaluate the prognosis of cancer patients. NLR, PLR MLR, GPS, and mGPS have been proved to be effective systemic inflammatory score indexes.

In this study, the prognostic value of SII in patients with gastric cancer was studied. SII is a systemic inflammatory index based on neutrophils, platelets, and lymphocytes. Neutrophils are mainly involved in nonspecific cellular immunity of the blood, which can release a large amount of nitric oxide, arginase, and reactive oxygen species (ROS), leading to disorders of T cell activation [24]. Circulating neutrophils have a prognostic value in a variety of tumors, and studies confirm that patients with larger peripheral neutrophilic granulocyte count, or NLR, have a lower survival rate [25, 26]. Platelets can protect the CTC from shear stress, induce epithelialmesenchymal transition, and promote the overflow of tumor cells to the metastatic site $[8,27]$. At the same time, it has been reported that platelets and neutrophils promote adhesion and spread in distant organs through secretion of vascular endothelial growth factor $[9,28]$. Lymphocytes are an important component of leukocytes produced in lymphoid organs, which are an important cellular component 
involved in the immune response. Lymphatic reflux in the body can make the lymph nodes receive a timely antigenic stimulation, secrete a large number of cells and humoural factors, generate specific immune response, and control tumor growth [29]. A decline in the number and function of lymphocytes will weaken immune surveillance and defence from cancer [29].

In this study, SII proved to be related to tumor location, histological grade, tumor size, TNM stage, and perineural infiltration in patients with gastric cancer and was an independent prognostic factor for patients with gastric cancer. SII has a better predictive ability than other existing prognostic indexes based on inflammation, such as NLR, PLR, and MLR. The nomogram established can accurately predict the 3- and 5-year survival rates of patients with gastric cancer after operation, and its accuracy is significantly higher than that of the $8^{\text {th }}$ edition AJCC staging system. There were some limitations in this study, such as selection bias and the retrospective study design. The systemic inflammatory response in the peripheral blood was not compared with local inflammation of tumors. However, the potent prognostic efficacy and convenient detection method of SII as a new systemic inflammatory score enable it to be beneficial. Many previous studies researched that systemic immune indexes can be used to predict efficacy of therapies in tumor patients [30-33], but we have only researched the relationship between the systemic immune indexes and gastric cancer patients' OS. We suggested further research on the relationship between systemic immune indexes and tumor relapse. In addition, the level of these indices in relapse cases and their dynamic changes with treatment response also need to be explored. In the future, a multicentre, prospective study is needed to verify our findings.

In conclusion, this study indicates that as a novel prognostic score based on inflammation, SII can independently predict the overall survival of patients with gastric cancer after operation, which is superior to the existing systemic inflammatory indexes (NLR, PLR, and MLR) that are based on peripheral blood immunity and inflammatory cells. The prognostic nomogram based on SII is a reliable model for predicting the postoperative survival of patients with gastric cancer.

\section{Data Availability}

The data used to support the findings of this study are available from the corresponding author upon request.

\section{Conflicts of Interest}

The authors declare that there is no conflict of interest regarding the publication of this article.

\section{Authors' Contributions}

All authors have read and approved the content and agreed to submit the article for consideration for publication in the journal. Hongtai Shi, Youqin Jiang, and Honggang Cao contributed equally to this work.

\section{References}

[1] J. Ferlay, I. Soerjomataram, R. Dikshit et al., "Cancer incidence and mortality worldwide: sources, methods and major patterns in globocan 2012," International Journal of Cancer, vol. 136, no. 5, pp. E359-E386, 2015.

[2] K. M. Fock, "Review article: the epidemiology and prevention of gastric cancer," Alimentary Pharmacology \& Therapeutics, vol. 40, no. 3, pp. 250-260, 2014.

[3] W. Chen, R. Zheng, P. D. Baade et al., "Cancer statistics in China, 2015," CA: A Cancer Journal for Clinicians, vol. 66, no. 2, pp. 115-132, 2016.

[4] R. L. Siegel, K. D. Miller, and A. Jemal, "Cancer statistics, 2016," CA: A Cancer Journal for Clinicians, vol. 66, no. 1, pp. 7-30, 2016.

[5] L. Huang, S. Liu, Y. Lei et al., "Systemic immune-inflammation index, thymidine phosphorylase and survival of localized gastric cancer patients after curative resection," Oncotarget, vol. 7, no. 28, pp. 44185-44193, 2016.

[6] Y. Shao, Y. Geng, W. Gu et al., "Assessment of lymph node ratio to replace the pn categories system of classification of the tnm system in esophageal squamous cell carcinoma," Journal of Thoracic Oncology, vol. 11, no. 10, pp. 17741784, 2016.

[7] D. Hanahan and R. A. Weinberg, "Hallmarks of cancer: the next generation," Cell, vol. 144, no. 5, pp. 646-674, 2011.

[8] M. Labelle, S. Begum, and R. O. Hynes, "Direct signaling between platelets and cancer cells induces an epithelialmesenchymal-like transition and promotes metastasis," Cancer Cell, vol. 20, no. 5, pp. 576-590, 2011.

[9] J. Cools-Lartigue, J. Spicer, B. McDonald et al., "Neutrophil extracellular traps sequester circulating tumor cells and promote metastasis," The Journal of Clinical Investigation, vol. 123, no. 8, pp. 3446-3458, 2013.

[10] A. Mantovani, P. Allavena, A. Sica, and F. Balkwill, "Cancerrelated inflammation," Nature, vol. 454, no. 7203, pp. 436444, 2008.

[11] C. Y. Ock, A. R. Nam, J. Lee et al., "Prognostic implication of antitumor immunity measured by the neutrophil-lymphocyte ratio and serum cytokines and angiogenic factors in gastric cancer," Gastric Cancer, vol. 20, no. 2, pp. 254-262, 2017.

[12] S. C. Wang, J. F. Chou, V. E. Strong, M. F. Brennan, M. Capanu, and D. G. Coit, "Pretreatment neutrophil to lymphocyte ratio independently predicts disease-specific survival in resectable gastroesophageal junction and gastric adenocarcinoma," Annals of Surgery, vol. 263, no. 2, pp. 292-297, 2016.

[13] Q. Deng, B. He, X. Liu et al., "Prognostic value of pre-operative inflammatory response biomarkers in gastric cancer patients and the construction of a predictive model," Journal of Translational Medicine, vol. 13, no. 1, p. 66, 2015.

[14] S. Li, X. Lan, H. Gao et al., "Systemic inflammation response index (siri), cancer stem cells and survival of localised gastric adenocarcinoma after curative resection," Journal of Cancer Research and Clinical Oncology, vol. 143, no. 12, pp. 24552468, 2017.

[15] B. Hu, X. R. Yang, Y. Xu et al., "Systemic immuneinflammation index predicts prognosis of patients after curative resection for hepatocellular carcinoma," Clinical Cancer Research, vol. 20, no. 23, pp. 6212-6222, 2014.

[16] B. L. Wang, L. Tian, X. H. Gao et al., "Dynamic change of the systemic immune inflammation index predicts the prognosis 
of patients with hepatocellular carcinoma after curative resection," Clinical Chemistry and Laboratory Medicine, vol. 54, no. 12, pp. 1963-1969, 2016.

[17] Y. Geng, Y. Shao, D. Zhu et al., "Systemic immuneinflammation index predicts prognosis of patients with esophageal squamous cell carcinoma: a propensity score-matched analysis," Scientific Reports, vol. 6, no. 1, article 39482, 2016.

[18] A. Passardi, E. Scarpi, L. Cavanna et al., "Inflammatory indexes as predictors of prognosis and bevacizumab efficacy in patients with metastatic colorectal cancer," Oncotarget, vol. 7, no. 22, pp. 33210-33219, 2016.

[19] X. Hong, B. Cui, M. Wang, Z. Yang, L. Wang, and Q. Xu, "Systemic immune-inflammation index, based on platelet counts and neutrophil-lymphocyte ratio, is useful for predicting prognosis in small cell lung cancer," The Tohoku Journal of Experimental Medicine, vol. 236, no. 4, pp. 297-304, 2015.

[20] R. L. Camp, M. Dolled-Filhart, and D. L. Rimm, "X-tile: a new bio-informatics tool for biomarker assessment and outcomebased cut-point optimization," Clinical Cancer Research, vol. 10, no. 21, pp. 7252-7259, 2004.

[21] F. Balkwill and A. Mantovani, "Inflammation and cancer: back to virchow?," The Lancet, vol. 357, no. 9255, pp. 539545, 2001.

[22] C. Maletzki and J. Emmrich, "Inflammation and immunity in the tumor environment," Digestive Diseases, vol. 28, no. 4-5, pp. 574-578, 2010.

[23] R. Duan and A. Nilsson, "Metabolism of sphingolipids in the gut and its relation to inflammation and cancer development," Progress in Lipid Research, vol. 48, no. 1, pp. 6272, 2009.

[24] I. Muller, M. Munder, P. Kropf, and G. M. Hansch, "Polymorphonuclear neutrophils and tymphocytes: strange bedfellows or brothers in arms?," Trends in Immunology, vol. 30, no. 11, pp. 522-530, 2009.

[25] M. Stotz, A. Gerger, F. Eisner et al., "Increased neutrophillymphocyte ratio is a poor prognostic factor in patients with primary operable and inoperable pancreatic cancer," British Journal of Cancer, vol. 109, no. 2, pp. 416-421, 2013.

[26] H. Cheng, F. Long, M. Jaiswar, L. Yang, C. Wang, and Z. Zhou, "Prognostic role of the neutrophil-to-lymphocyte ratio in pancreatic cancer: a meta-analysis," Scientific Reports, vol. 5, no. 1, article 11026, 2015.

[27] D. Schumacher, B. Strilic, K. K. Sivaraj, N. Wettschureck, and S. Offermanns, "Platelet-derived nucleotides promote tumor-cell transendothelial migration and metastasis via p2y $\mathrm{y}_{2}$ receptor," Cancer Cell, vol. 24, no. 1, pp. 130-137, 2013.

[28] S. Najmeh, J. Cools-Lartigue, R. F. Rayes et al., "Neutrophil extracellular traps sequester circulating tumor cells via $\beta 1$ integrin mediated interactions," International Journal of Cancer, vol. 140, no. 10, pp. 2321-2330, 2017.

[29] C. Ferrone and G. Dranoff, "Dual roles for immunity in gastrointestinal cancers," Journal of Clinical Oncology, vol. 28, no. 26, pp. 4045-4051, 2010.

[30] L. Rossi, M. Santoni, S. J. Crabb et al., "High neutrophil-tolymphocyte ratio persistent during first-line chemotherapy predicts poor clinical outcome in patients with advanced urothelial cancer," Annals of Surgical Oncology, vol. 22, no. 4, pp. 1377-1384, 2015.

[31] A. Farolfi, M. Petrone, E. Scarpi et al., "Inflammatory indexes as prognostic and predictive factors in ovarian cancer treated with chemotherapy alone or together with bevacizumab. A multicenter, retrospective analysis by the Mito group (Mito 24)," Targeted Oncology, vol. 13, no. 4, pp. 469-479, 2018.

[32] C. Lolli, U. Basso, L. Derosa et al., "Systemic immuneinflammation index predicts the clinical outcome in patients with metastatic renal cell cancer treated with sunitinib," Oncotarget, vol. 7, no. 34, pp. 54564-54571, 2016.

[33] C. Lolli, O. Caffo, E. Scarpi et al., "Systemic immuneinflammation index predicts the clinical outcome in patients with mcrpc treated with abiraterone," Frontiers in Pharmacology, vol. 7, p. 376, 2016. 


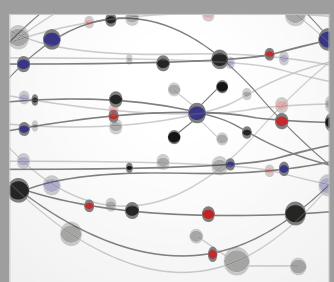

The Scientific World Journal
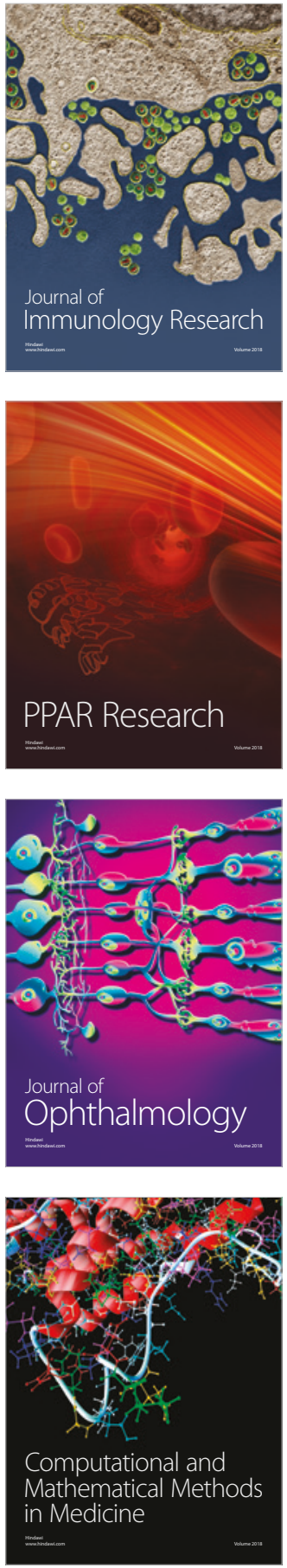

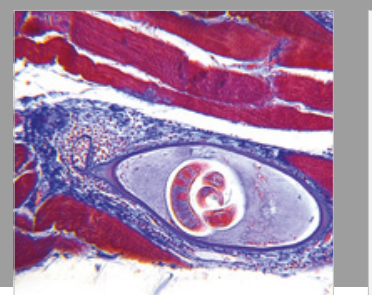

Gastroenterology Research and Practice

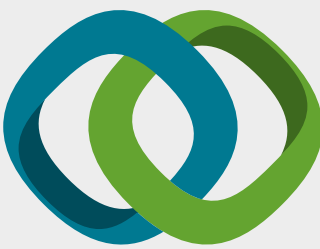

\section{Hindawi}

Submit your manuscripts at

www.hindawi.com
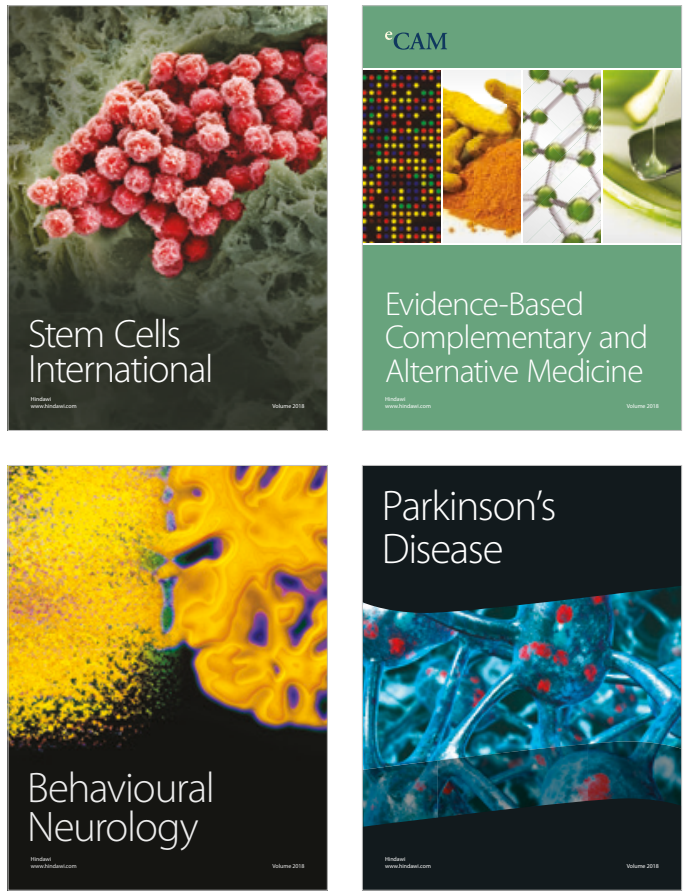

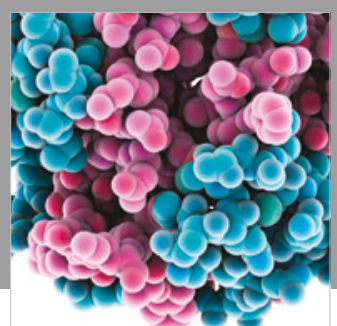

ournal of

Diabetes Research

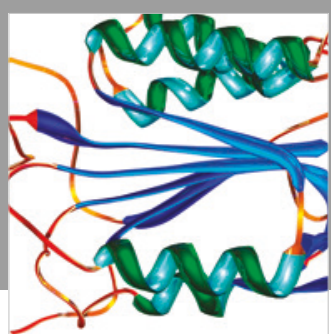

Disease Markers
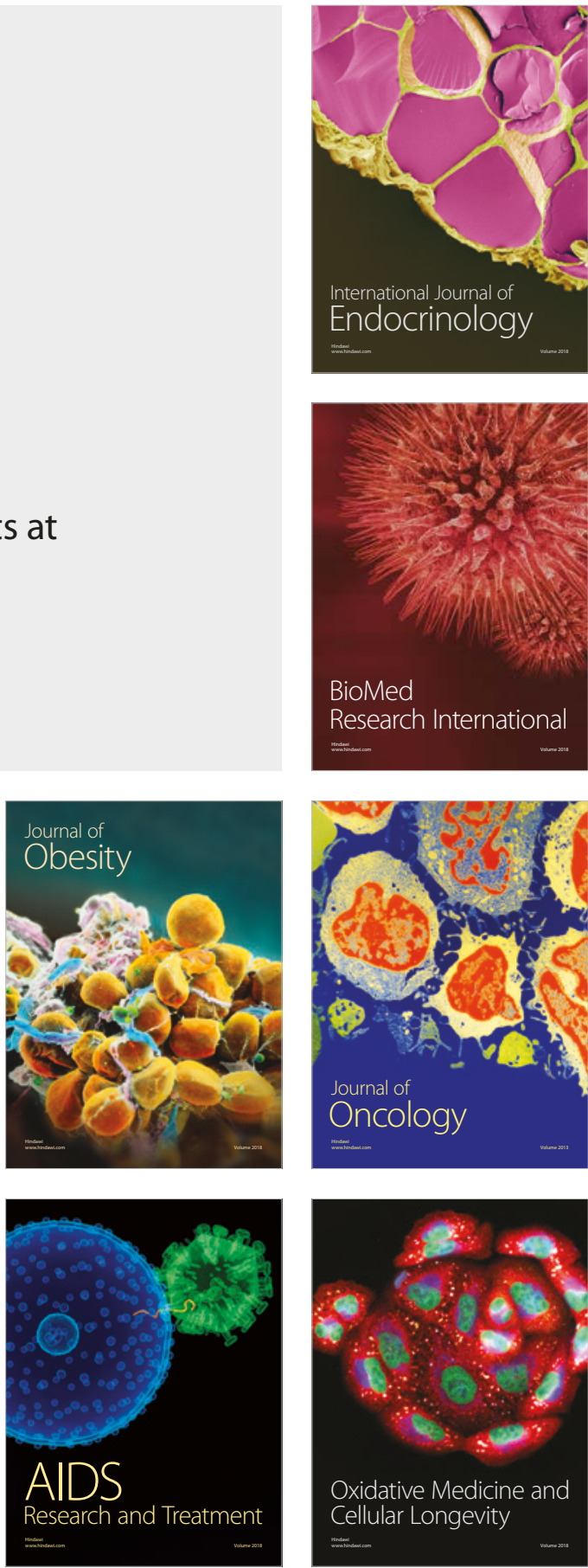